\title{
Micro-Rhetoric in Dialogic Interaction
}

(1.1) Oh! I'm invited to a wedding that night. But the bride is pregnant so I might drop by in the wee hours

The piece of discourse in (1.1) is taken from the facebook event page for a birthday party. The person who wrote (1.1) on the wall of the event did so to communicate that she would be busy on the night of the party, but that she might be able to stop by anyway. Many of us might find her communication clear and her reason for possibly being able to stop by quite reasonable. However, if we think of the argumentation in the second part of the example it is not obvious how the discourse coheres:

(1.2) The bride (of the wedding I am going to) is pregnant, so I might drop by (at the party) in the wee hours.

Because of the conventional implicature generated by "so", we recognise (1.2) as an argument. By "argument" we mean a piece of discourse where some proposition is supported or explained by another proposition. However, for this argument to be successful - in the sense that the host of the birthday party understands and accepts that the bride being pregnant is a good reason for the guest to be able to stop by at the birthday party later on - additional information is required. This additional information might be a chain of inference like "if the bride is pregnant, she will be tired", and "if she is tired the wedding might not go on for that long".

Moreover, it seems to be the case that-faced with a discourse like (1.1), which conveys an argument - a language user may tentatively accommodate some warrant which would underpin the argument. Arguments like these, which require additional world knowledge to be acceptable or understandable, are important in rhetoric where they are called enthymemes. Crucial for the use of enthymemes in rhetorical discourse is that they are based on principles or notions which are so obvious to the audience that the argument seems to express necessity, rather than possibility. The basis for this seems to be cognitive - if we have to do less work to understand an argument, the content of it will appear more natural to us.

Walker (1996) suggests that this is also true for dialogue: Presenting a proposition in connection with some support or back up facilitates processing even 
when the supporting proposition does not add new information. Walker gives numerous examples that can be seen as constituting arguments with implicit premises.

Enthymemes are not uncommon in conversation. They seem to play a role over and above the rhetorical device found in speeches, and enthymeme-like inferences are at the heart of theories of implicature-not least Relevance Theory (Sperber and Wilson, 1995; Wilson and Sperber, 2004). Despite this, very little work has been done on enthymemes as such in dialogue. An exception to this is Jackson and Jacobs (1980) who link the phenomenon to general principles of conversation.

This work aims to remedy this situation by placing the enthymeme in the context of interactional linguistics and pragmatics. We propose an account of how enthymemes in dialogue interact with patterns of reasoning stored in our cognitive resources to provide structure in discourse and communicate implicit information. Such patterns, in rhetorical theory, are referred to as topoi. We also suggest how enthymemes and topoi can be included in a dialogue semantic program, extending theories like KoS (Ginzburg, 2012), where TTR, a type theory with records (Cooper 2005a, 2012) is used to capture dialogue phenomena unaccounted for by traditional formal approaches. In this chapter we will first look at some fields of research in linguistics and computational linguistics which provide many of the main ideas of the theory presented in this book. We will then provide some brief background on enthymemes and topoi and their role in interaction, and finally sketch an outline of the book.

\subsection{Interaction Based Linguistics}

Consider the interpretation of rise in (1.3):

(1.3) CHERRILYN: Yeah I mean $\langle$ pause $\rangle$ dog hairs rise anyway so

FIONA: What do you mean, rise?

CHERRILYN: The hair $\langle$ pause $\rangle$ it rises upstairs.

(BNC file KBL:42O1-4203)

A snippet of dialogue such as (1.3) can be difficult to make sense of, and this difficulty lies in determining the meaning of particular lexical items (such as rise), but also - perhaps to a greater degree - in building hypotheses about situational and discursive contexts where the exchange in (1.3) would make sense. If we consider a larger excerpt from the same dialogue (1.4), we get a better idea 
of what is going on. From an analytical point of view, however, describing in a precise way how a speaker of English makes sense of (1.4) is challenging to say the least.

(1.4) CHERRILYN: Most dogs aren't allowed up 〈pause $\rangle$ upstairs.

He's allowed to go wherever he wants $\langle$ pause $\rangle$ do whatever he likes.

FIONA: Too right!

So they should!

Shouldn't they?

CHERRILYN: Yeah I mean 〈pause > dog hairs rise anyway so

FIONA: What do you mean, rise?

CHERRILYN: The hair 〈pause $\rangle$ it rises upstairs.

I mean I, you know friends said it was, oh God I wouldn't allow mine upstairs because of all the 〈pause $\rangle$ dog hairs!

Oh well pause $\rangle$ they go up there anyway.

FIONA: So, but I don't know what it is, right, it's only a few bloody hairs!

(BNC file KBL:4196-4206)

The dialogue in (1.4) is an excerpt from a conversation regarding dogs (which we will consider in more detail in Chapter 6), and whether or not they should be allowed in certain parts of the house, particularly upstairs. This overarching content is something that most people who have a moderate knowledge of English could determine in a few seconds after first seeing the text. However, the interpretation process is complex, and accounting for it involves explaining many diverse phenomena: We need to explain how we manage to interpret the contextual meaning of words, which is sometimes - as in the case of rise in (1.4) - ambiguous, and we need a theory for how words are combined to express propositions. However, we also need to account for how utterances make sense in relation to other utterances. This includes things like anaphor resolution-how do we define, for example, to whom "they" in "Oh well 〈pause〉 they go up there anyway" refers?

We also need to explain how speakers make inferences that are necessary for the dialogue to cohere. For example, how does Cherrilyn's utterance about what her friend said serve to address Fiona's clarification request What do you mean, rise? And how do we relate Fiona's final utterance So, but I don't know what it is, right, it's only a few bloody hairs! to the rest of the dialogue?

Traditional semantics tends to ignore the complexity demonstrated above and instead treats language in terms of sentences which are generated via a set 
of rules pertaining to an ideal speaker, and the context-free truth conditions of these sentences (Montague, 1973; Kratzer and Heim, 1998).

In pragmatics the truth-conditional accounts of meaning are extended to things like presupposition and implicature. In more recent approaches some of these problems are addressed and to some extent resolved, in the sense that context is taken into account when meanings are interpreted. However, typical dialogue features such as non-sentential utterances (Fernández and Ginzburg, 2002), cross-person compound contributions (Howes, 2012), and disfluencies (Clark and Fox Tree, 2002) are still largely ignored. So, if we are going to analyse language as it appears in dialogue, it would entail additional complexity. For example, some dialogue contributions are in fact non-sentential, a fact that has been noted by philosophers such as Wittgenstein (1953) as well as by linguists doing data driven research (Fernández and Ginzburg, 2002; Schlangen and Lascarides, 2003; Fernández et al., 2007).

Moreover, research in psychology as well as Conversation Analysis (CA) has established that dialogue participants easily interpret and produce incomplete utterances, that is, utterances which are interrupted or where the speaker stops mid sentence (Goodwin, 1979; Altmann and Kamide, 1999). Since conversation is such a essential part of language use, trying to understand how language works without taking dialogue phenomena into account means we will not have a complete picture.

\subsubsection{Dialogism}

One interesting aspect of the excerpt in (1.4) is that the dialogue does not run completely smoothly. Cherrilyn says something that Fiona obviously does not understand, and so Fiona makes a clarification request. The fact that we as language users sometimes fail to correctly interpret an utterance provides some clues to what it is that we do when our interpretation is actually successful. How we manage to set the conversation straight again, how we correct our mistakes, is revealing.

One of the approaches to linguistics that emphasises the importance of dialogue is the dialogical tradition originating in the ideas of Bakhtin (1986). The umbrella of dialogism covers various types of research, philosophical as well as empirical. Common traits in dialogical research are contextualism and interactionism. Contextualism means that contexts are always viewed as relevant, and in fact a primary factor in communication. Interactionism means that dialogue, considered as a kind of interaction between agents, is central to the understanding of how language works. One aspect of this is a focus on features typical of dialogue like repairs, corrections and co-constructed sentences. 
Linell (1998, 2009) — one of the most recent representatives of the dialogistic tradition-contrasts the dialogistic and the monologistic view, which (in the case of linguistics and similar fields) is characterised by a conception of interaction as secondary in the understanding of communication. Linell concedes that a certain amount of monologistic analysis is necessary in linguistic research, as in other research. However, he is skeptical of the reductionism in formal theories. The risk, according to Linell, is that theories which do not pay sufficient attention to the interactive aspect of language, tend to give their users a distorted picture of how language actually works.

Since the late nineties, however, a branch of formal semantics has emerged which takes interaction as its point of departure, and in fact shares many assumptions about the nature of language with dialogism. One notable representative of this line of research is Ginzburg, who has been developing his program — notably in his book The Interactive Stance (Ginzburg, 2012)—over the last decades.

\subsubsection{Dialogue Semantics}

Influenced by insights from philosophy, artificial intelligence and conversation analysis Ginzburg (2012) has developed KoS, a semantic framework which combines insights from Conversation Analysis and dynamic semantic approaches like Discourse Representation Theory (DRT, Kamp, 1981; Kamp and Reyle, 1993) and Segmented Discourse Representation Theory (SDRT, Asher and Lascarides, 2003; Lascarides and Asher, 2008).

KoS offers a way of accounting for misunderstandings and miscommunication using dialogue gameboards (DGB) cast in TTR-a type theory with records first introduced by Cooper (2005b,a, 2012) - to represent the development of the dialogue participants' information states through the course of a dialogue.

One of the fundamental assumptions underpinning any type of dialogue semantics is that structure in language is to a great extent created through interaction - a viewpoint which formal dialogue theories like KoS have in common with Conversation Analysis. Many of the phenomena addressed in work in the KoS spirit involve issues that are relevant to this kind of structure, such as questions, interjections and non sentential utterances, repair and context. Out of these issues, the ones that are most obviously related to enthymemes and topoi are dialogical structure-creating phenomena and context.

In theories of rhetorical relations such as Rhetorical Structure Theory (RST, Mann and Thompson, 1986, 1988) and SDRT (Asher and Lascarides, 2003) a finegrained taxonomy of rhetorical relations has been proposed, of which many are not dialogical per se, even though they do occur in dialogue. Examples of such relations are background, narration and contrast. 
The approach taken in this book can be said to be a version of KoS. However, the formal details are closer to TTR as it appears in the work of Cooper (2012, 2016) and Breitholtz and Cooper (2011). We will take a closer look at some of the relevant similarities and differences in Chapters 3 and 4.

\section{Micro-rhetorical Linguistics}

The interest in "imperfect" language use is something that Ginzburg and Linell have in common with Paul Hopper. In his paper Linguistics and Micro-Rhetoric: A Twenty-First Century Encounter, (Hopper, 2007), he argues that grammar is essentially an abstraction of the way we string together prefabricated fragments and fixed phrases, and that grammaticality as well as deviations from grammaticality can usually be explained by our tendency towards efficiency in communication - which is emphasised in rhetoric. Hopper argues that the interactive perspective with its focus on language as situated in time and space brings the disciplines of linguistics and rhetoric closer together:

... in fact (usage based) linguistics is nothing but the micro-end of rhetoric HOPPER (2007) p. 236

Hopper argues that a micro-rhetorical analysis would differ from the type of analysis usually applied in traditional rhetoric - if we want to find out things about words and phrases rather than debates, speeches and their contexts and effects, we have to look at smaller bits of language like utterances or short episodes. The linguistic phenomena which Hopper is interested in are mainly syntactic, like the case of apo koinou (or pivot) constructions, sometimes found in natural data. Apo koinou is a construction where one constituent serves as the end of one grammatical sentence and the beginning of another, for example "That's what grabs their attention most is adverts" (Hopper, 2007).

However, if we apply a micro-rhetorical perspective to other areas of linguistics, there seem to be other language phenomena which are to some extent addressed in rhetoric, often from a different perspective than that applied to the same phenomena in linguistics. In semantics and pragmatics for example, the notion of inference is essential. In semantics we study inferences like presuppositions, which derive from the meanings of particular words and constructions. For example the word "again" in a sentence such as "I lost the book again", triggers the presupposition that the speaker has lost the book before. This is due to the meaning of "again", and not context dependent. 
Other types of inferences, like conversational implicatures, are to a great extent dependent on context and on the assumption of some general expectations we have about communication. Inferences are also central in rhetoric. In fact, the art of rhetoric is much focused on how to lead an audience to make particular inferences, and thereby become convinced of the point which the speaker wishes to communicate.

In rhetorical theory the enthymeme is the type of evidence, or proof, which relates to reasoning and making inferences. In the early eighties, Jackson and Jacobs (1980) suggested that enthymemes are related to conversational practices that we use continuously when we talk and communicate in other ways. They argue that the rhetorical enthymeme is derived from more general principles of communication and interaction. While this seems likely, it is nevertheless the case that the connection between inferences and conversational phenomena such as turn-taking and preference structure has not been a focus in linguistics. Inferences like implicature and presupposition are mainly studied in philosophy of language and pragmatics, while conversational practices and contextualised language use is mainly considered by conversation analysts. However, in rhetoric there is a focus on the inferential quality of language in use as well as on how we should use this quality to our advantage, that is, how we should employ inferences to make the communication run smoothly in a particular context.

It seems to us that the rhetorical perspective has not been considered enough in linguistics although it could contribute to the understanding of inference, coherence and other phenomena in dialogue and other types of linguistic communication. Thus, we aim in this work to look more closely at enthymematic reasoning and how it plays out in dialogue. In Section 1.6 we will state our aim in more detail, but let us first take a closer look at the concepts of enthymeme and topos.

\subsection{The Aristotelian Enthymeme}

\subsubsection{Aristotelian Rhetoric}

Aristotle's Rhetoric was intended as instruction on the art of public speaking, but in fact it is also a comprehensive introduction to a number of aspects of linguistics which are relevant to the study of linguistic interaction. It does not deal with logic, but with the logic-like type of reasoning which frequently occurs in dialogue and other types of discourse. Aristotle's Rhetoric also discusses emotions and their causes and effects, as well as cognitive aspects of language and style. Thus Aristotelian rhetorical theory actually combines ele- 
ments of what we would today call the pragmatics, psycholinguistics and sociolinguistics of dialogue.

For a modern day researcher who looks to the classics, it is important to know whether one is motivated by an interest in historical reconstruction, i.e. in trying to understand a text in its historical context and interpreting its original meaning, or in attempting to seek inspiration from the insights of classical theorists. Since our aim here is to use our interpretation of some Aristotelian notions to contribute to contemporary theories of dialogue semantics and pragmatics, this work is clearly a case of the latter. Therefore we will just give a brief account of the social and cultural context of the Rhetoric, and of the concepts of enthymeme and topos as they occur in renditions of Aristotle's text. ${ }^{1}$

Aristotle's Rhetoric was written as a guide for students of rhetoric in a context where the ability to speak well in public was important to any free citizen. There were no professional lawyers or prosecutors, so anyone who wanted to take a case to court or who was summoned to court to meet an accusation had to stand up and speak for himself (for a more detailed account, see Corbett and Connors, 1999). Also, in a democracy like Athens where ordinary people without much insight into public matters and state affairs were allowed to vote, it was essential for anyone aspiring to a political career to master the art of persuasion by referring to the likelihood of possibilities rather than to knowledge and facts alone. In the Rhetoric, book one, chapter one, section eleven, Aristotle explicitly states the importance of tapping into common beliefs and opinions when dealing with a crowd, rather than presenting the audience with facts and strictly logical reasoning:

Speech based on knowledge is teaching, but teaching is impossible with some audiences; rather, it is necessary for pisteis (proof) and speeches as a whole to be formed on the basis of common beliefs.

Rhet I 1.11

\subsubsection{The Syllogism and the Enthymeme}

Apart from the importance of adjusting the arguments to the audience, Aristotle claims that rhetoric should not (as it had in previous rhetorical handbooks) focus on external matters such as the different parts of a speech and in which order they should appear in the discourse, but rather on logos, reasoning (Rhet. I 1.9), which is also the aspect of rhetoric which is most relevant to our discussion.

1 All citations of the Rhetoric refer to Kennedy's translation (Aristotle, ca. 34 O B.C.E./2007). 
Before we look further at Aristotle's view of rhetorical reasoning, let's say something about his view of the related field of logic. First, it is important to remember that Aristotelian logic is not equivalent to modern, formal logic, although it has been pointed out that the differences are perhaps less pronounced than what was thought to be the case in the early zoth century (Smith, 2012). One essential difference, however, is that while modern logic is formulated by means of artificial languages, Aristotle dealt only with natural language. A central concept in the Aristotelian theory of deduction is the syllogism. In the Prior Analytics Aristotle defines it as

An argument (logos) in which, certain things having been supposed, something different from the things supposed results of necessity because these things are so

$\operatorname{Pr}$ An I 1.24b 18-20

The phrase "certain things having been supposed" refers to the set of premises, and, being in the plural, "the things supposed" indicates, according to Keyt (2009), that there must be more than one premise. In rhetoric, the correlate of the deductive type of proof, the syllogism, is the enthymeme. The conclusion of an enthymeme does not - in contrast to that of a syllogism - need to follow of necessity. Nor is there a need for the set of premises to consist of more than one premise. These are formal requirements, but there are also some other ways in which syllogisms and enthymemes differ, mainly having to do with subject matter. While logical arguments should deal with general statements, enthymemes deal with particular cases. Thus, while in logic you argue for or against a general claim about the world, in rhetoric you seek to persuade someone of something regarding a particular case.

In (1.5) we see an example of a syllogism, where the conclusion necessarily follows from the premises. In (1.6) on the other hand, the conclusion depends on a notion that if someone has done something which is considered bad, then that person is likely to also have done less bad things. As Aristotle puts it: "if the greater thing is true, then the lesser is also, for people strike their fathers less than their neighbours" (Rhet. II 23.4).

(1.5) Socrates is a man

All men are mortal

Socrates is mortal

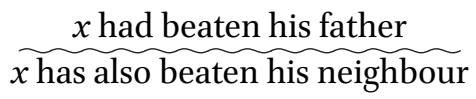


Presenting an argument based on implicit premises is possible since the members of an audience-just like people who partake in a conversationhave knowledge and beliefs regarding the world around them. In this case Aristotle expected the audience to recognise, based on experience and previous input, that it is more common and a lesser crime to beat your neighbour than your father, so when they hear that someone is known to have beaten his father, they may find it quite reasonable that he is also guilty of beating his neighbour, if such charge has been made against him.

\subsection{Topoi-the Warrants of Enthymemes}

In order to be efficient, an enthymeme needs to draw on some commonly recognised notion that "fills in" the information that is lacking in the set of premises. This notion Aristotle refers to as the topos of the enthymeme. Some topoi may be applied to various subjects, while others are specific to a particular subject. An example of a general topos is the topos of the more and the less, of which Aristotle says

... to form syllogisms or speak enthymemes from this about justice is just as possible as about physics or anything else, although these subjects differ in species.

Rhet I 2.21

An example of a general type of topos is that of opposites, on which the enthymeme in (1.7) is based.

(1.7) a. ... to be temperate is a good thing, for lack of self-control is harmful (Rhet II 23.1.)

b. lack of self-control is harmful

The enthymeme in (1.7) draws on the idea that since self-control and lack of self-control are opposites, the opposite of what is true of self-control is true of lack of self-control. Alternatively, and more generally, if two things are opposites, the opposite of what is true of the first must (or is usually) true of the second. This example shows that the common notion of Aristotelian enthymemes as syllogisms with one hidden or silent premise, is not always correct. Strictly speaking, enthymemes based on the topos of opposites seem to require a set of additional premises to constitute a reasonable argument to an audience. 


\subsubsection{Topoi in Linguistics}

The concept of topos is essential in the theory of argumentation presented by Ducrot $(1980,1988)$, to an extent in collaboration with Anscombre (1995). The theory is based on the idea that between two utterances $U$ and $V$ where one of them is an assertion or a suggestion, exhortation, etc. and the other an assertion which functions as a support for the first, there is always a link which sanctions the interpretation of $U$ and $V$ as an argument. For example, imagine a situation where two people are at the cinema trying to decide which film to see. One of them utters $(1.8)$, where $(1.8 \mathrm{~b})$ is clearly a reason for the suggestion made in (1.8a).
a. $A$ : Let's not see a drama
b. $A$ : I'm too tired

According to Ducrot a dialogue contribution like (1.8) exploits a link which sanctions the interpretation that the drama genre should be avoided by tired people. This link could be the idea that dramas are complex and cognitively challenging compared to other genres. This seems reasonable and something that most adults would recognise, if not agree with. A link like this is referred to by Ducrot and Anscombre as a topos.

Ducrot (1988) argues that topoi are notions which are common, that is they are assumed or taken for granted in a community, even before the conversation in which they are employed takes place. Topoi are also gradual, that is if I say "it's warm today, let's go to the beach", the topos - that warm weather makes the beach an attractive destination - is more true the warmer it is, and less true the less warm it is. A consequence of this would be that an enthymeme evoking a topos may be more or less convincing, depending on the context of utterance.

Topoi are also general in the sense that one topos can be employed in various arguments, in various situations. The opposite, that different topoi may be employed in similar situations, is also true. Anscombre (1995) argues that when we say Give a coin to the porter, he carried the bags all the way here, there is an obvious connection between the first and second proposition expressed in the utterance. However, the connection between "carrying luggage" and "getting a tip" is not linguistic, it's the common place principle that work should be rewarded, which is generally recognised, if not agreed upon in all situations. Interestingly, argues Anscombre, there are other, equally acceptable, principles which would lead to an opposite conclusion, such as principles that porters get paid to carry luggage already, and you should not get a tip for doing your job.

Anscombre also makes the important observation that topoi, contrary to logical rules, do not constitute a monolithic system. Instead the system of 
topoi consists of principles which may be combined in different ways, like logical rules, but which may be inconsistent if combined in a specific situation. Anscombre (1995) suggests that this is because topoi are part of ideology, ways in which we perceive the world, and ideologies are not monolithic. Therefore, a principle like opposites attract and birds of a feather flock together may co-exist not only in one community, but in the set of topoi of one individual, and be applicable in different contexts.

\subsubsection{Topoi as Cultural Indicators}

The idea of a dichotomy of beliefs in episteme and doxa goes back to Plato. On the Platonic view, episteme is knowledge about the world of forms (world of ideas), while doxa represents our beliefs about the world of perception.

The concept of doxa has been connected to rhetoric in an interesting way. Rosengren (2002) argues that doxa is in some respects a more relevant concept than episteme: The difference, as Rosengren sees it, between traditional epistemology and the doxology he argues in favour of, is that while epistemology is concerned with (universally) true beliefs, doxology is concerned with what is held to be true, appropriate or right in a certain context-in other wordswhich topoi are applicable in that context. To be aware of what is consistent with the doxa - the topoi- of a certain community could thus be more relevant for a speaker constructing arguments than the beliefs which are actually true-it is possible to construct convincing arguments from false beliefs just as well as from true ones.

The technique of adapting your arguments to the beliefs of the audience is well established in rhetorical theory, and Rosengren argues that rhetoric can be seen not only as a tool for forming arguments which are persuasive to a certain audience in a certain context, but also as a tool for finding out what the speaker and the audience of a discourse believe to be true (or, in the case of the audience, what the speaker believes they believe to be true), right and just.

Rosengren follows Perelman and Olbrechts-Tycteca (1969) in declaring that the topoi which a speaker's arguments draw on to some extent define the world view of the speaker and the addressee. Rosengren (2002, p. 87), argues that in this terminology, it is possible to describe different societies by describing the topoi which are dominant within these societies. In the context of dialogue modelling, this would mean that modelling the topoi available to an agent is a way of modelling that agent's take on (a limited part of) the agent's sociocultural context. 


\subsection{Linking Enthymeme and Topos}

To conclude the discussion about the view of enthymemes and topoi in previous literature, we should say something about the role they play in discourse and in relation to each other.

Enthymemes are units of discourse that may simultaneously serve many purposes. Enthymemes convey propositions that are not explicit in the discourse. They do this by requiring underpinning by topoi for an acceptable interpretation. The pragmatic meaning conveyed by an enthymeme in relation to a listener depends on which topos the listener accesses in the interpretation process.

In (1.9) President Bush, in his "State of the Union" address 2005, argues that Americans must join together to save social security, since the system is headed towards bankruptcy (the enthymeme $\varepsilon$ below). This argument is underpinned by a topos ( $\tau$ below) that if a certain state of affairs is beneficial or good, it should be safeguarded. The argument also depends on a premise that social security is a good thing.

(1.9) a. "... we must join together to strengthen and save Social Security" (Bush, 2005 State of the Union par. 16).

"[Because] Social Security will be paying out more than it takes in ... by the year 2042, the entire system would be exhausted and bankrupt." (Bush, 2005 State of the Union par. 19).

b. $\varepsilon=\underbrace{\text { the social security system is heading towards bankruptcy }}_{\text {we must save social security }}$

c. $\tau=\underbrace{\text { something worth keeping is heading towards destruction }}_{\text {it must be saved }}$

If we accept the validity of $\tau$ in (1.9c), and (at least) the premise that social security is something worth keeping, the conclusion of $\varepsilon$ in (1.9b) follows by necessity. It is thus possible to accept the topos, but not the premises that make the enthymeme an instantiation of the topos, and thus reject the enthymeme. 


\subsection{Aim and Outline of This Book}

In this chapter we have described a number of dialogue features where enthymematic structure seems to play a role. Examples of such features are sensemaking and coherence in language use. These questions are at the heart of pragmatics, and much of what we refer to here as "enthymematic reasoning" is discussed in theories of implicature, such as Gricean and neo-Gricean approaches (Horn, 1984; Levinson, 2000) and Relevance Theory (Sperber and Wilson, 1995; Carston and Hall, 2012). Some of the processes described in this book are also accounted for in other theories. However, while theories of implicature account for many of the principles of pragmatic reasoning, they still rely heavily on inferential processes which are not defined in the theory. Drawing on principles such as the principle of relevance and Gricean maxims we can make predictions about the existence of implicated conclusions, but we cannot make any precise predictions about the nature of these conclusions given a certain situational context and background. The theory presented here can make such predictions, which makes it possible to use in implementations of pragmatic inference.

We also considered a number of approaches to linguistics which we believe share many theoretical and methodological assumptions. All of these-dialogism, KoS and the micro-rhetorical perspective-focus on accounting for phenomena which are common in dialogue. Coherence in conversation cannot always be accounted for by theories of anaphora and other phenomena treated in dynamic semantics. We often express ourselves elliptically, engage in clarification, and repair our utterances. Adopting a theoretical standpoint where these features are relevant parts of language rather than nonstandard features, means among other things that context and pragmatic processes must be integrated, or at least possible to integrate, in any linguistic theory.

Enthymematic reasoning relies heavily on context, and often interacts with dialogue features such as clarification and ellipsis. Our aim is thus to formulate a theory for how enthymemes and topoi play a role in dialogue, in a framework that allows for a rich account of context as well as integration of dialogue features such as repair and clarification.

In the remainder of this book we will first, in Chapter 2, look at a number of linguistic phenomena discussed in the literature which are in various ways related to enthymemes. In Chapter 3 we will give a brief introduction to TTR and information states modelled as gameboards, and sketch a gameboard analysis of some simple dialogue examples involving enthymematic reasoning. In Chapter 4 we will go into the types of information states in more detail and 
extend the model by introducing update rules to account for interpretation of dialogue contributions involving enthymemes and topoi. In Chapter 5 we will consider how dialogue participants draw on topoi in inventing enthymemes and how the production and interpretation of enthymemes are related to particular conversational games. In Chapter 6 we will look at some applications of our theory, considering issues like non-monotonic reasoning and lexical disambiguation. Finally, in Chapter 7 , we will present our conclusions and discuss remaining problems and future work. 